\title{
Register nominum ad Vol. XXXVIII
}

Actis Dato, A., Brusca, A., Levi, V., und Solerio, F., 53 Amorim, D. de S., and Burchell, H. B., 319 Arnold, E. F., et Maurice, P., 85 (S) -, v. Ferrero, C.

Bertelli, G., v. Caniggia, A. Blondeau, Ph., v. Dubost, Ch. Brom, A. G., v. Desbaillets, P.

Brozek, J., v. Simonson, E.

Brusca, A., v. Actis Dato, A.

Bühlmann, A., v. Lichtlen, P.

-, v. Uehlinger, A.

Burchell, H. B., v. Amorim, D. de S.

Caniggia, A., Bertelli, G., and

Lodone, M., 371 Cleempoel, H., v. Polis, O. Condorelli, M., v. Sciacca, A. Cottier, P., 389 (B)

394 Register nomin

Desbaillets, P., Rivier, J. L.,

Mahaim, Ch., Brom, A. G., Livio, J. J., et Saegesser, F., 152 (S)

Doret, J. P., v. Ferrero, C.

Dubost, Ch., Blondeau, Ph., et Piwnica, A., 158 (S)

Ferrero, C, Arnold, E. F., et Doret,

J. P., 80 (S) Fleisch, A. O., 172 (S)

Gasser, P., v. Jost, M.

Gloor, R., 296

Grauwiler, J., und Jasiński, B., 288

Grewin, K. E., 1

Grosse-Brockhoff, F., 142 (S)

Hegglin, R., und Rutishauser, W.,

249 -, v. Rutishauser, W. Heinecker, R., 219 (B) Herbinger, W., 267 Hochrein, H., v. Schneider, K. W. Hoványi, M., v. Unghváry, L.

Jasiński, B., v. Grauwiler, J. Jost, M., und Gasser, P., 215 (S)

Kappert, A., 389 (B) Kriehuber, E., v. Wenger, R.

Levi, V., v. Actis Dato, A. Lichtlen, P., Bühlmann, A., und

Schaub, F., 197 (S) Linhart, J., and Prerovský, I., 21 Livio, J. J., v. Desbaillets, P. Lodone, M., v. Caniggia, A. Lüthy, E., und Rutishauser, W.,

183 (S) -, v. Rutishauser, W.

Mahaim, Ch., v. Desbaillets, P. Massie, E., and Walsh, T. J., 390 (B) Maurice, P., v. Arnold, E.

F. Moesslacher, E., v. Wenger, R. Müller, P., 90 (S)

Nieveen, J., Slikke, L. B. van der, Sien, Que Giok, and Vries, Hk. de, 239

Oka, M., and Savola, P., 279

ad Vol. XXXVIII

Pippig, L., v. Schneider, K. W. Piwnica, A., v. Dubost, Ch. Polis, 0., et Cleempoel, H., 335

Prerovský, I., v. Linhart, J. 
Rentsch, M., v. Stampbach, O.

Reubi, F, 390 (B)

Reutter, F., Schaub, F., und

Uehlinger, A., 190 (S) Rivier, J. L., v. Desbaillets, P. Rutishauser, W., Lüthy, E., und

Hegglin, R., 101 (S) -, v. Hegglin, R. -, v. Lüthy, E.

Saegesser, F., v. Desbaillets, P. Savola, P., v. Oka, M. Sciacca, A., and Condorelli, M., 389 (B) Schaub, F., v. Lichtlen, P. -, v. Reutter, F.

Schmitt. O. H., v. Simonson, E. Schneider, K. W., Hochrein, H., und

Pippig, L., 205 (S) Scholer, H., 66 (S) Schüpbach, P., v. Stampbach, O. Schweitzer, P., 223 Sien, Que Giok, v. Nieveen, J. Simonson, E., Brozek, J., and

Schmitt, O. H., 258 Slikke, L. B. van der, v. Nieveen, J. Solerio, F., v. Actis Dato, A. Stampbach, O., Wyler, F., Rentsch,

M. und Schüpbach, P., 112 (S)

Uehlinger, A., und Bühlmann, A.,

357 -. v. Reutter, F. Unghváry, L. und Hoványi, M., 31

Vries, Hk., de, v. Nieveen, J.

Walsh, T. J., v. Massie, E. Wenger, R., Moesslacher, E., und

Kriehuber, E., 37 Whiteside, C. W., 222 (B) Wolf, H. J., 63 (B), 221 (B) Wuhrmann, F., 221 (B)

Wyler, F., v. Stampbach, O.

Zeisler, E. B., 220 (B) 